\title{
Attention to configural information in change detection for faces
}

\section{Simone K Favelle}

School of Psychology, University of Wollongong, Northfields Avenue, Wollongong, NSW 2522, Australia; e-mail: simone_favelle@uow.edu.au

\section{Darren Burke}

Centre for the Integrative Study of Animal Behaviour (CISAB), Macquarie University, Sydney, NSW 2169, Australia

Received 22 September 2005, in revised form 29 November 2006; published online 10 September 2007

\begin{abstract}
In recent research the change-detection paradigm has been used along with cueing manipulations to show that more attention is allocated to the upper than lower facial region, and that this attentional allocation is disrupted by inversion. We report two experiments the object of which was to investigate how the type of information changed might be a factor in these findings by explicitly comparing the role of attention in detecting change to information thought to be 'special' to faces (second-order relations) with information that is more useful for basic-level object discrimination (first-order relations). Results suggest that attention is automatically directed to second-order relations in upright faces, but not first-order relations, and that this pattern of attentional allocation is similar across features.
\end{abstract}

\section{Introduction}

The human face is a complex visual stimulus that conveys important social and biological information. Not surprisingly then, detecting and/or identifying a face is rapid and may even be mandatory; however, current evidence suggests that these processes require at least some attentional resources (see Palermo and Rhodes 2007 for a review of face perception and attention). But what properties within a face attract our attention? Many studies have focused on investigating the different facial regions (eg the eyes, mouth) that might be more salient or attract our attention (Barton et al 2003; Davies and Hoffman 2002; Gosselin and Schyns 2001; Sergent 1986; Shepard et al 1981). However, it has been argued that what sets face processing apart from other object processing is the reliance on the configuration of features (Diamond and Carey 1986).

Configural processing can be thought of as involving either first-order or secondorder relations [or holistic processing, see Maurer et al (2002) for a review]. All faces have a similar layout of features: two laterally separated eyes above a centrally placed nose above a centrally placed mouth. This layout comprises the first-order configural relations of a face. Within this particular layout, individual faces differ in the spacing or distance between features. These distances are referred to as second-order spatial relations. Because all faces share the same first-order relations, second-order relational information is required to individuate faces. The importance of second-order relations for face processing has been demonstrated in a number of studies. Discrimination of faces that differ in second-order relations is much more difficult in inverted faces than in upright faces, a discrepancy that is not found for faces that differ in features (Friere et al 2000; Leder and Bruce 2000; Leder et al 2001; Malcolm et al 2004; Searcy and Bartlett 1996). That is, second-order relations are easily processed in upright faces, but are difficult to extract from inverted faces. In addition, when fine-detailed featural information is removed from a face by blurring the stimulus whilst leaving the second-order relations intact, identification accuracy is not impaired (Hayes 1988; 
Sergent 1986). Inverting blurred faces severely impairs accuracy, presumably because the inversion disrupts the processing of second-order relations (Collishaw and Hole 2000; Schwaninger et al 2002).

As these studies suggest, it is sensitivity to these second-order spatial relations between the parts of the face on which our ability to recognise individual faces depends (and to which 'configuration' usually refers). Whether this sensitivity to second-order relations is due to practice (Diamond and Carey 1986; Gauthier and Tarr 1997; Gauthier et al 1999), or the fact that faces automatically activate a brain region that is particularly tuned to configurational differences (Farah 1996), is a matter of considerable, ongoing debate. A second, less studied, issue is whether the configural sensitivity is a consequence of attentional or perceptual factors. Recent studies by Davies and Hoffman (2002) and by Barton and colleagues (Barton et al 2003; Malcolm et al 2004) have used the change-detection paradigm along with cueing tasks to investigate the way in which attention is allocated to various types of information and regions within a face.

Davies and Hoffman (2002) used a flicker task to study detection of changes to the eyes or mouth of unfamiliar faces, with configural (second-order) and featural changes examined separately across these different facial regions. In line with previous research, Davies and Hoffman found an inversion effect for configural changes to both the eyes and the mouth. However, in contrast to what is typically found in recognition and other face-processing tasks, they also found an inversion effect for local feature changes to both the eyes and the mouth. For both experiments, Davies and Hoffman used a cueing task (in which a word indicating a facial feature cued the location of change) as a perceptual control. They reasoned that, if perceptual rather than attentional impairments were responsible for the inversion effect, cueing should not affect change-detection performance for inverted faces. Results of the perceptual control task of local changes in both upright and inverted faces showed that subjects detected change faster and more accurately in cued than in uncued faces. A similar pattern was found for configurally different faces, although the trend was not significant in the accuracy data. Further, significant interactions between part and cue suggested that performance improved more for cued changes to the mouth than for cued changes to the eyes. Davies and Hoffman's main conclusions were that the eyes receive greater attention than the mouth and that upright faces receive more efficient attention than inverted faces.

Barton et al (2003) investigated the interaction between attention and salience in face processing. Specifically, they compared attention to the eyes and the mouth using a change-detection paradigm, and found that subjects were best able to detect changes to eye position (eyes moved inwards 8 pixels each), next best at detecting changes to eye colour (lightening) or mouth position (moved upwards 8 pixels), and worst at detecting changes to mouth colour. Inversion of the faces impaired detection of mouth position changes much more than other kinds of changes, and cueing the kind of change improved detection performance for all changes but eye position. These results are consistent with the idea that subjects naturally allocate more attention to eye position than to the other aspects manipulated (and so cueing has no effect on detecting such changes), and with the allocation of attention being impaired by face inversionespecially attention to the mouth. Together with earlier results that showed that increasing viewing time or focusing attention to the lower face reduced the inversion impairment for mouth position changes (Barton et al 2001), Barton and colleagues argue that the regional variation in the face-inversion effect is based on differences in the salience of regions across the face.

The configural changes used by both Davies and Hoffman (2002) and Barton et al (2003) were changes to the second-order relations of the face. In fact, most of the 
evidence for configural effects in face perception/recognition (especially those of the effect of inversion) comes from studies that manipulate second-order spatial relations (eg Friere et al 2000; Haig 1984; Hosie et al 1988; Kemp et al 1990; Leder et al 2001). An exception is Malcolm et al (2004) who investigated whether the type of spatial relation change (first-order or second-order) was a factor in the regional variation in the face-inversion effect found previously by Barton and colleagues. Malcolm et al showed that, while first-order and second-order changes are equally detectable in upright faces, inversion impairs detection of changes to the mouth more than to the eyes for second-order changes, but the effect of inversion is similar in detection of changes to the mouth and eyes for first-order changes. That is, whether the face is upright or inverted, first-order changes are perceived nearly as well in the lower as in the upper part of the face.

As Maurer et al (2002) point out, humans are remarkably sensitive to the firstorder relations of faces, even in the absence of normal facial features. First-order relations may be important for face detection or basic-level object categorisation, whereas second-order relations appear to be important for within-class discrimination (Cooper and Wojan 2000; Gauthier et al 1998). So, when engaged in a lower-level face-processing task such as change detection, do we selectively attend to second-order relations (thought to be 'special' for face processing) over first-order relations and featural information (thought to be more important for basic-level categorisation)? Recent research by Barton and colleagues (Barton et al 2003; Malcolm et al 2004) suggests that this may be the case; however, as far as we are aware no research to date has explicitly compared the effects of attention on first-order versus second-order configural information.

Change detection is commonly studied with either a flicker task or a one-shot task. A flicker task involves alternating an original and modified image repeatedly, separating the images with a brief blank screen, until observers detect the change. A one-shot task is similar, but involves only one alternation of the original and modified images. The one-shot task minimises the involvement of eye movements and long-term memory (ie is based on short-term representations), while the flicker task rules out the possibility that the inability to detect change is due to memory consolidation failure (Rensink 2002). It is argued that change detection depends on visual attention being focused on the region of change (Rensink 2000, 2002; Rensink et al 1997, 2000; Wolfe 1999), where visual attention is defined as a limited-capacity mechanism used to select certain types of visual information for further processing at the expense of other visual information. Thus, determining the kinds of changes that are successfully detected in a flicker or one-shot task will reveal the kinds of information that are more readily attended. We intend to use a flicker task to investigate detection of changes to different types of information available in faces, to establish which of these different aspects of a face attract focused attention. The results of Barton and colleagues (Barton et al 2001, 2003; Malcolm et al 2004) suggest that attention depends on the region of change; specifically, the eyes receive more attention than the mouth. However, we wish to compare directly detection of first-order and secondorder changes as well as changes to the eyes, nose, and mouth in upright and inverted faces to determine which of these different types of information are more readily attended.

Another technique for investigating attention is the cueing paradigm. Attention may be shifted by using an accurate cue to the location of change, producing a benefit (relative to no cue) in performance. If attention is naturally or more efficiently allocated to a particular aspect (eg layout, configuration, features, colour, etc) of a face or scene, then cueing should have no effect on performance (Aginsky and Tarr 2000). 
Davies and Hoffman (2002) found that cueing was more effective for featural changes than for configural changes, which they explained in terms of configural-change detection approaching ceiling levels of performance. However, another interpretation of this result is that the type of cue used was of greater benefit to featurally changed faces than configurally changes faces. Davies and Hoffman used a centrally presented word cue (identifying a facial feature) that was $100 \%$ reliable when there was a change. ${ }^{(1)}$ This type of cueing could force participants to process the faces in a localised or piecemeal fashion, rather than in a more natural, holistic manner. For example, if the cue was 'eyes', the participant needs only encode information about the eyes to be able to either (i) successfully detect change or (ii) successfully judge that no change had occurred. Thus, while a local encoding strategy would be useful for featural change, the encoding of more global information would be needed to represent configural information.

In the second experiment, we employed invalid-cue and neutral-cue conditions as well as valid-cue conditions. Contrary to the above studies, the cue (a centrally presented word identifying a facial feature) was not $100 \%$ reliable and change could involve either first-order or second-order configural information; thus, participants would not benefit from a local encoding strategy. Further, in order to fully investigate the role of attention in face processing, it is important to examine the effect of drawing attention away from a particular aspect or region of the face as well as when we move attention toward an aspect or region (Posner et al 1978).

The aim of the current study is to use the change-detection paradigm to investigate the effect of selective attention on configural information that is 'special' to face processing (ie second-order relations), and how this compares to the effect of attention on information that is not 'special' to face processing (ie first-order relations information). We make a direct comparison of sensitivity to first-order and second-order configural changes in upright and inverted faces. In particular, we investigate (i) whether there is differential performance for detection of change for different types of information in a flicker task; (ii) whether the effect of cueing in a one-shot task is similar for detecting changes to second-order relations and first-order relations; and (iii) whether inversion influences the effect of cueing. Further, we wish to examine whether any of these effects of attention or type of information processing depend on the facial region involved in the change.

\section{Experiment 1: Flicker task}

We employed a flicker task to investigate the locus of attention in face processing, the rationale being that the changes that are detected reflect the information that is more readily attended. Detecting changes to a face is a task that does not require processing the stimuli as faces, and so it may be better able to tap early visual processing of faces than a recognition task. Because changes can occur to any part of the face, and to any aspect of the configuration, there is also no incentive for subjects to allocate their attention strategically, which makes it likely that the results will reflect 'natural' attentional allocation. We expect that inversion will significantly disrupt sensitivity to second-order configural relations (eg Friere et al 2000; Leder and Bruce 2000; Leder et al 2001), but the relative degree to which first-order configural relations are affected is unknown. On the basis of the results of Malcolm et al (2004; their experiment 2), we expect the effect of inversion for second-order changes to be greater for the mouth than eyes, whereas the effect of inversion for first-order relation changes should be similar for both eyes and mouth.

(1) All trials were cued; however, half of the trials involved change ('different' trials) and half did not ('same' trials). Thus, the cue was $100 \%$ reliable if there was a change, but on half the trials the cued region did not change (because nothing changed). 


\subsection{Method}

2.1.1 Participants. Twenty-three undergraduate students from the University of Wollongong participated in the experiment. None had participated in any of the other experiments reported in this paper. All had normal or corrected-to-normal vision.

2.1.2 Materials. A set of ten digitised (256 grey levels, 106 dots per inch) Caucasian faces in a full-face pose with a neutral expression was used as stimuli. For each face in this set (five female and five male), nine foil faces were generated with the Adobe Photoshop graphics package. Foil faces were created by making first-order relation and second-order relation changes to each of the eyes, nose, and mouth facial features (see figure 1). ${ }^{(2)}$ First-order changes involved moving: (i) one eye up or down 16 pixels (half of the faces had the left eye moved, the other half the right, and half had the eye moved up, the other half down); (ii) the nose left or right 16 pixels (half of the faces left, half right); and (iii) the mouth left or right 16 pixels (half of the faces left, half right). Second-order changes involved moving: (i) both eyes; (ii) the nose; and (iii) the mouth, up or down 16 pixels. Half of the faces had a left moved feature, half right. Note that the foil face differed from the target face only in respect of the feature and change type that was being tested-everything else was held constant.

The target faces were approximately $8 \mathrm{~cm} \times 11 \mathrm{~cm}$ when displayed on a 15 inch monitor (screen size, $832 \times 624$ pixels at 72 pixels per inch) and were viewed from a distance of approximately $70 \mathrm{~cm}$ (subtending an approximate visual angle of $6.5 \mathrm{deg}$ $\times 9 \mathrm{deg}$ ). The stimulus presentation was controlled with RSVP (www.tarrlab.org) on a Power Macintosh 7200.

2.1.3 Procedure and design. We used a $2 \times 2 \times 3$ repeated-measures design including the factors of orientation (upright and inverted), change type (first-order and second-order relations), and facial feature (eyes, nose, and mouth). Half of the trials had upright face displays, half inverted. Half of the trials were 'same' trials and half 'different'. The 'different' trials were split equally into the nine conditions. ${ }^{(3)}$ Thus, the experiment consisted of 360 randomly ordered trials $(2 \times 3 \times 3 \times 2 \times 10$ faces $)$. The experimental trials were preceded by 5 practice trials.

All stimuli were shown at the centre of the screen. Each trial began with a fixation cross appearing for $1500 \mathrm{~ms}$, followed by the first (standard) face, followed by a mask, followed by a second face which was also followed by a mask. This sequence was repeated 7 times. Each face appeared for $250 \mathrm{~ms}$, and the mask for $100 \mathrm{~ms}$. The trial timed out after $8000 \mathrm{~ms}$. The next trial began $1000 \mathrm{~ms}$ after the subject made a response or the trial timed out. Participants were asked to make a two-alternative forced-choice decision indicating whether the two alternating face images were the 'same' or 'different' by pressing appropriate keys on a keyboard.

(2) An additional set of foils was created by changing the shape of components and these were included in both of the current experiments. Component shape changes involved changing the shape of each facial feature by means of the filter functions in Adobe Photoshop. For example, lips and noses were made thinner and wider or fatter, eyes were made rounder or smaller. Importantly, the original facial features only were modified, thus there were no colour or texture changes that could potentially act as additional cues to the change. However, performance was at or below chance for a substantial number of the conditions that included this type of change, possibly owing to the more subtle nature of the component shape change than used in previous studies (Barton et al 2003 used a brightness manipulation and Davies and Hoffman 2002 used a Thatcherisation of features). Thus, the component-shape condition was dropped entirely from this study.

${ }^{(3)}$ Including the above-mentioned component shape-change condition, which was subsequently omitted from analysis. 

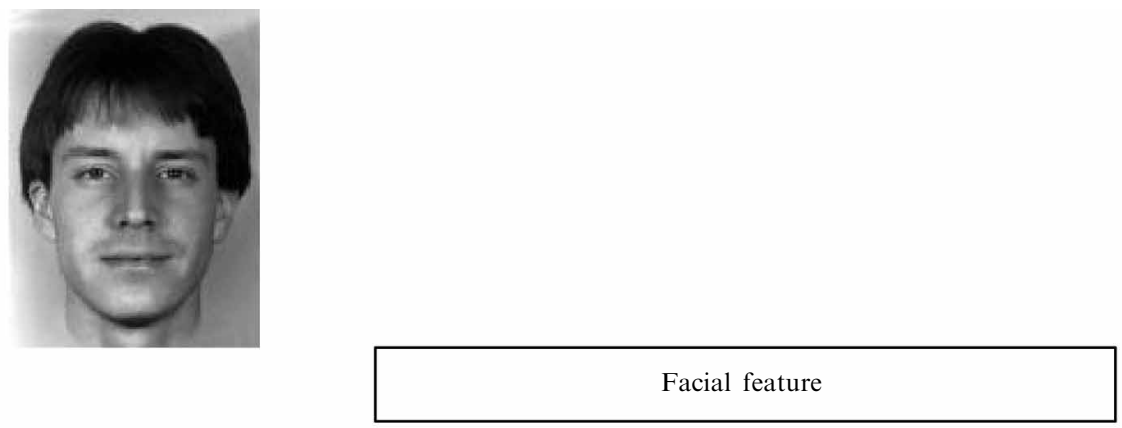

eyes

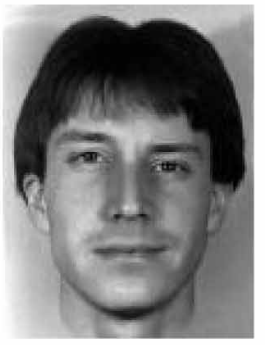

first-order configural relations
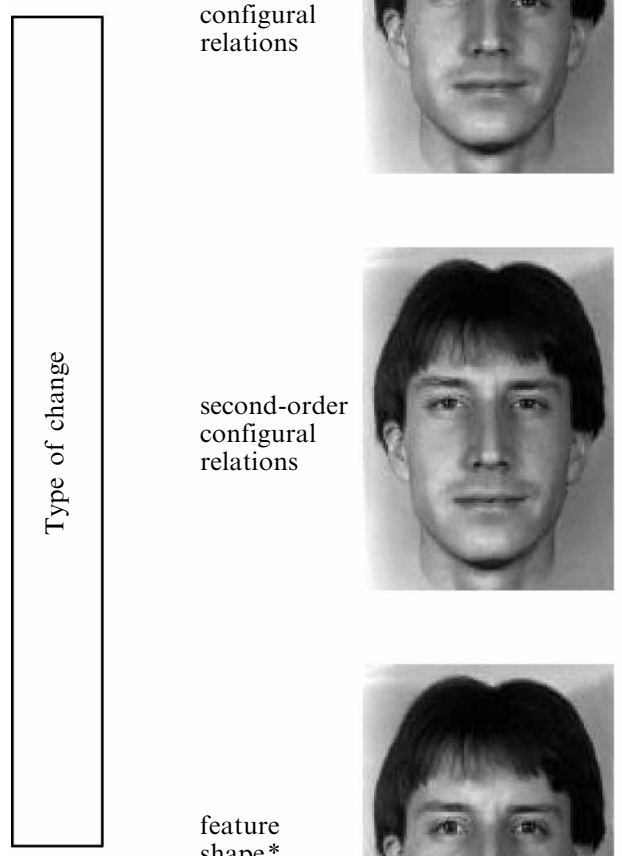

second-order configural relations
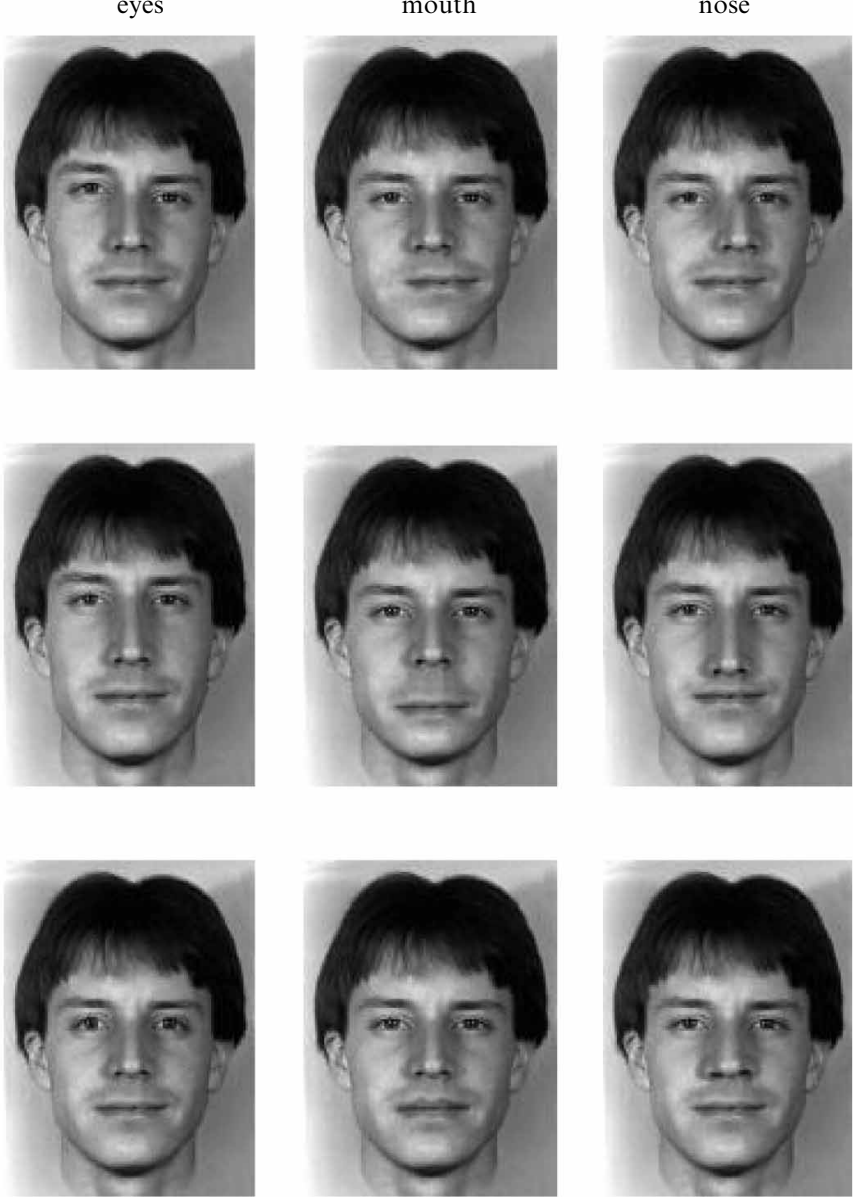

Figure 1. Examples of the face stimuli. Nine foils were generated for each original face by making first-order relation, second-order relation, and component shape changes to the eyes, nose, and mouth. * The component shape change was omitted from analysis, see text for details.

\subsection{Results and discussion}

Two separate three-way $(2 \times 2 \times 3)$ repeated-measures ANOVAs including the withinsubjects factors of orientation (upright and inverted), change type (first-order and second-order relations), and facial feature (eyes, nose, and mouth) were conducted on accuracy (proportion correct) and response time (RT) data. Trials for which no response was made were excluded from data analysis. 
The three-way repeated-measures ANOVA conducted on the mean proportion correct data revealed significant main effects of orientation $\left(F_{1,22}=55.69, p<0.01\right.$; upright: mean $(\mathrm{M})=0.84, \mathrm{SE}=0.016$; inverted: $\mathrm{M}=0.62, \mathrm{SE}=0.022$ ) and change type $\left(F_{1,22}=57.67, p<0.01\right.$; first-order relations: $\mathrm{M}=0.84, \mathrm{SE}=0.017$; second-order relations: $\mathrm{M}=0.62, \mathrm{SE}=0.022)$, but not facial feature $(p=0.18)$. All of the two-way interactions and the three-way interaction between orientation, facial feature, and change type were significant $(p<0.05)$.

Orientation interacted with facial feature $\left(F_{2,44}=10.08, p<0.01\right)$. As can be seen in figure 2, the effect of inversion was much greater overall for changes to the mouth and eyes than for changes to the nose, which showed very little impairment due to inversion. Orientation also interacted with change type $\left(F_{1,22}=14.41, p<0.01\right)$. Again, looking at figure 2, there was a larger inversion effect for second-order than firstorder changes $(p>0.05)$. The difference in accuracy between upright and inverted was nearly twice as large for second-order changes $(0.29)$ than first-order changes $(0.16)$.

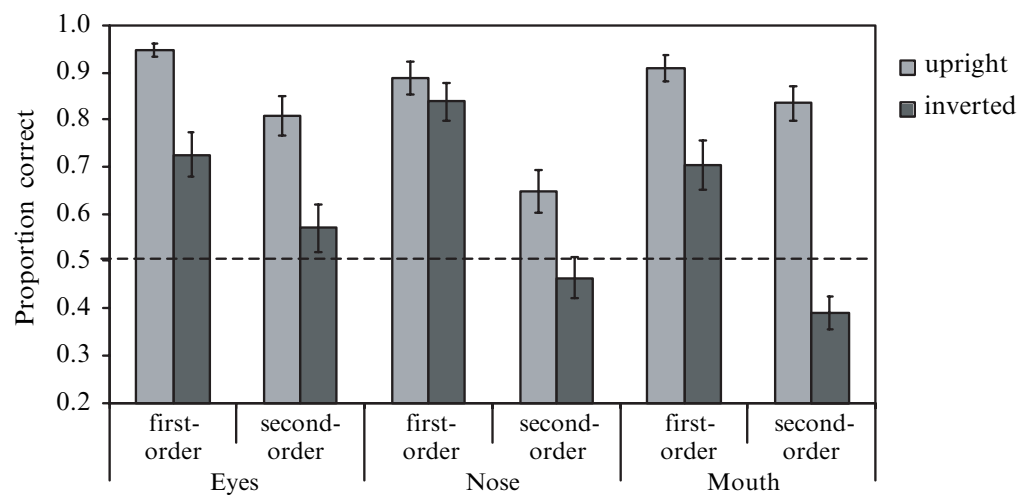

Figure 2. Results of experiment 1. Mean upright and inverted proportion-correct scores are shown as a function of facial feature, change type, and orientation. Error bars represent standard errors of the mean. The dashed line here and in subsequent figures indicates chance $(0.5$ proportion correct).

The two-way interaction between facial feature and change type $\left(F_{2,44}=10.04\right.$, $p<0.01)$ appears to be due to there being little difference in change-detection performance between the three facial features for first-order relation changes, yet secondorder relation changes to the eyes are detected with relatively greater accuracy than to the mouth or nose. However, there is a significant three-way interaction between orientation, facial feature, and change type $\left(F_{2,44}=3.74, p=0.03\right)$ reflecting the proportionately larger inversion impairment for second-order changes to the mouth than for any other feature or change type, and the relative lack of an inversion effect for changes made to the nose. This finding is very much in line with that of the studies of Barton and colleagues on the regional variations in face processing and the inversion effect.

Only reaction times for correct trials were analysed. This resulted in a number of empty cells in the RT data owing to some subjects recording zero accuracy in some conditions. In total there were 2 missing RT values $(0.7 \%$ of total data cells) in two different conditions, each of which was replaced with the mean for that condition. Generally speaking, the RT data reflected the results found for the accuracy data. The three-way repeated-measures ANOVA conducted on the mean proportion-correct data revealed significant main effects of orientation $\left(F_{1,22}=26.78, p<0.01\right.$; upright: $\mathrm{M}=1208, \mathrm{SE}=56$; inverted: $\mathrm{M}=1490, \mathrm{SE}=72)$ and change type $\left(F_{1,22}=20.04\right.$, $p<0.01$; first-order relations: $\mathrm{M}=1205, \mathrm{SE}=53$; second-order relations: $\mathrm{M}=1495$, $\mathrm{SE}=74$; shape $\mathrm{M}=1846, \mathrm{SE}=170)$, but not facial feature $(p=0.2)$. 


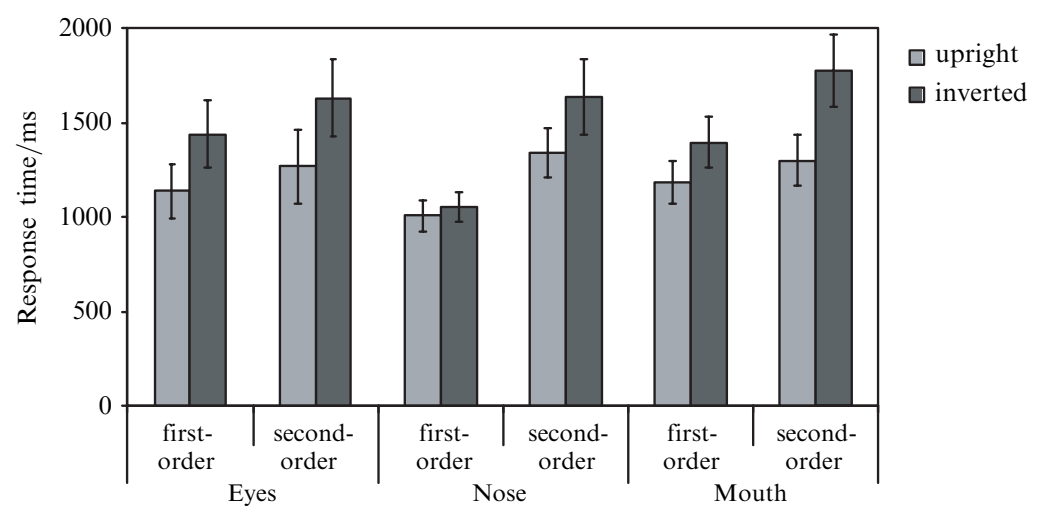

Figure 3. Results of experiment 1. Mean response times to upright and inverted faces are shown as a function of facial feature, change type, and orientation. Error bars represent standard errors of the mean.

The interaction between orientation and change type was significant $\left(F_{4,88}=3.63\right.$, $p<0.01)$. While there is little difference in RT to detect changes to second-order information between the three facial features, change detection is relatively faster (i) for the eyes than for the mouth or nose for shape changes, and (ii) for the nose than for the eyes or mouth for first-order changes. The interaction between orientation and change type was approaching significance $\left(F_{1,22}=4.24, p=0.052\right)$. As seen in figure 3 , the change-detection RT for second-order changes is impaired by inversion, whereas inversion has a much smaller effect on RT for first-order changes. The interaction between orientation and feature was not significant $(p=0.23)$, nor was the three-way interaction between orientation, facial feature, and change type $(p=0.48)$.

To the extent that change-detection performance reflects the distribution of visual attention, attention to facial features differs with face orientation and the type of change that occurs. Attention to the nose was much less affected by inversion than attention to the eyes or mouth and attention to second-order information regarding the mouth was most severely affected by inversion, in line with findings by Barton and colleagues (Barton et al 2001, 2003). Participants were effectively 'blind' (performed at or below chance level) to second-order changes in inverted faces. This suggests two things. First, although first-order changes are easier to detect in upright than in inverted faces, they are detected reasonably well in inverted faces. That is, first-order information is not as sensitive to inversion as the other changes. Second, in conditions similar to those in which we normally individuate people, ie when faces are upright, second-order changes are detected well, but inverting the faces severely disrupts this process.

\section{Experiment 2: Cued one-shot task}

In experiment 2 we investigated detection of changes to first-order and second-order configural relations in upright and inverted faces using valid, invalid, and neutral cues to direct attention in a one-shot change-detection task. Experiment 2 was the same as experiment 1, except that only one alternation was viewed (that is, participants saw an initial face, followed by a mask, then the second face) and we had valid-cue, invalid-cue, and neutral-cue conditions. Malcolm et al (2004) also used a one-shot change detection task; however, they used cues that were $100 \%$ valid. An advantage of the cueing design used in experiment 2 is that it does not encourage observers to process faces in a piecemeal fashion, since (i) the cue does not always 
accurately indicate the region of change, and (ii) the change could involve first-order or second-order relations.

\subsection{Method}

3.1.1 Participants. Thirty-one undergraduate students from the University of Wollongong participated in the experiment. None had participated in experiment 1. All had normal or corrected-to-normal vision.

3.1.2 Materials. The stimuli were a subset of those used for experiment 1. Two male and two female faces were chosen from the set of ten used in the previous experiment.

3.1.3 Procedure and design. We used a $2 \times 3 \times 3 \times 2$ repeated-measures design including the factors of orientation (upright and inverted), cue (neutral, valid, and invalid), facial feature (eyes, nose, and mouth), and change type (first-order relations and second-order relations). Half of the trials had upright-face displays, half inverted. Half of the trials were 'same' trials and half 'different'. Thus, the experiment consisted of 432 randomly ordered trials $(2 \times 3 \times 3 \times 3 \times 2 \times 4$ faces $){ }^{(4)}$ The reliability of the cues was not equal; $25 \%$ of cued trials had a valid cue to the location of change whereas the remaining $75 \%$ of cued trials had either an invalid cue to the location of change or were 'same' trials (in which there was a cue but no change).

Each trial began with a fixation cross appearing for $500 \mathrm{~ms}$ at the centre of the screen. In the valid-cue and invalid-cue conditions, the words "eyes", "nose", or "mouth" appeared after the fixation cross for $1000 \mathrm{~ms}$. The fixation cross remained on screen for $1000 \mathrm{~ms}$ in the neutral-cue condition. The cue (word or fixation cross) was followed by the first (standard) face for $1500 \mathrm{~ms}$, immediately followed by a mask appearing on the screen for $500 \mathrm{~ms}$, and finally the second face which remained on the screen until the subject responded or the trial timed out after $5000 \mathrm{~ms}$. Each face was randomly placed at a position 25 pixels in any direction from the centre of the screen. The next trial began $1000 \mathrm{~ms}$ after the subject made a response or the trial timed out. Participants were asked to make a two-alternative forced-choice decision indicating whether the two alternating face images were the 'same' or 'different' by pressing appropriate keys on a keyboard.

\subsection{Results and discussion}

A $2 \times 3 \times 2 \times 3$ repeated-measures ANOVA including the within-subjects factors of orientation (upright and inverted), facial feature (eyes, nose, and mouth), change type (first-order and second-order), and cue (neutral, valid, and invalid) was conducted on accuracy (proportion correct) and response time (RT) data. Trials for which no response was made were excluded from data analysis.

The four-way repeated-measures ANOVA conducted on the mean proportion correct data revealed significant main effects of orientation $\left(F_{1,30}=109.36, p<0.01\right.$; upright: $\mathrm{M}=0.91, \mathrm{SE}=0.008$; inverted: $\mathrm{M}=0.65, \mathrm{SE}=0.013)$, facial feature $\left(F_{2,60}=24.21\right.$, $p<0.01$; eyes: $\mathrm{M}=0.83, \mathrm{SE}=0.012$; mouth: $\mathrm{M}=0.79, \mathrm{SE}=0.015$; nose: $\mathrm{M}=0.72$, $\mathrm{SE}=0.016)$, change type $\left(F_{1,30}=60.87, p<0.01\right.$; first-order relations: $\mathrm{M}=0.85, \mathrm{SE}=$ 0.010 ; second-order relations: $\mathrm{M}=0.71, \mathrm{SE}=0.013)$, and cue $\left(F_{2,60}=12.98, p<0.01\right.$; neutral cue: $\mathrm{M}=0.78, \mathrm{SE}=0.014$; valid cue: $\mathrm{M}=0.82, \mathrm{SE}=0.013$; invalid cue: $\mathrm{M}=0.74, \mathrm{SE}=0.016$ ).

Change-detection accuracy for features differed as a function of the orientation of the face $\left(F_{2,60}=6.18, p<0.01\right.$, see figure 4$)$. The effect of inversion on changes to the mouth was greater than the effect of inversion on changes to the eyes or nose (which is consistent with Barton et al 2003, and Davies and Hoffman 2002). 

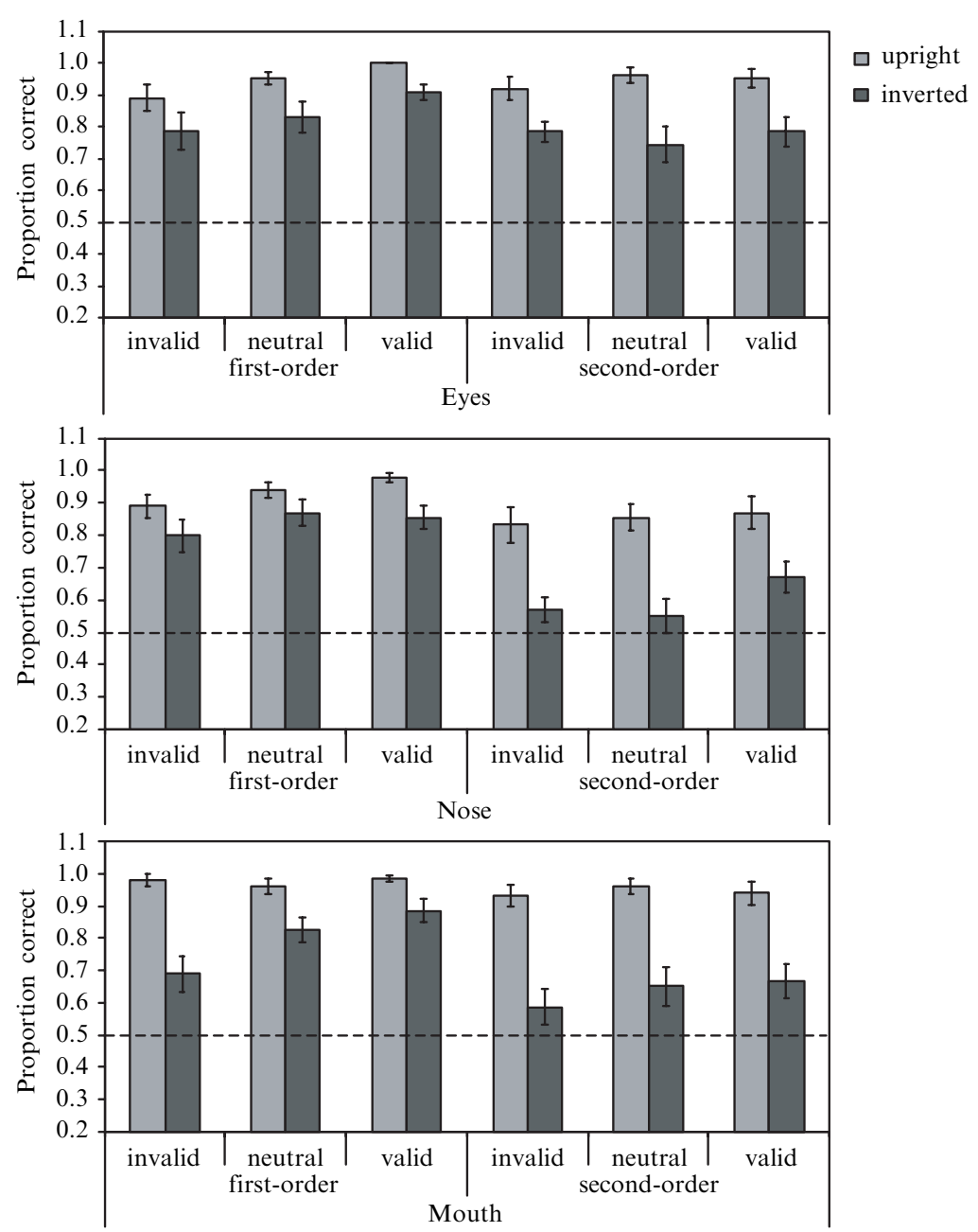

Figure 4. Results of experiment 2. Mean upright and inverted proportion-correct scores are shown as a function of cue, change type, and orientation for each of the three facial features tested. Error bars represent standard errors of the mean.

Orientation also interacted with change type $\left(F_{1,30}=28.27, p<0.01\right)$. The effect of inversion was far greater for second-order relation changes than for first-order ones (see figure 4). Bonferroni adjusted a posteriori comparisons show that there was no significant difference between first-order and second-order change-detection accuracy for upright faces $(p=0.22)$, but that second-order changes in inverted faces were significantly harder to detect than in upright faces $(p<0.01)$. Change type interacted with facial feature $\left(F_{2,60}=8.67, p<0.01\right)$. While first-order changes were detected equally well across all features (all $p$ s $>0.17$ ), second-order changes to the eyes were detected more accurately than second-order changes to either the mouth or the nose (both $p \mathrm{~s}<0.05$ ). There was a significant three-way interaction between orientation, change type, and facial feature $\left(F_{2,60}=4.80, p=0.01\right)$ which appears to be due to the differences between features being much more pronounced for second-order changes to inverted faces than for any other change type and orientation combination.

Cue interacted with change type $\left(F_{2,60}=4.32, p=0.02\right)$. The effect of cueing was greater for first-order changes than for second-order changes. Indeed, cueing appeared to have little effect at all on second-order changes. Bonferroni adjusted a posteriori comparisons showed that for second-order changes there was no significant difference 
between invalid and neutral cues or valid and neutral cues (both $p$ s $>0.19$ ). However, in line with the pattern seen in traditional cueing studies (eg Posner et al 1978) firstorder changes were detected more accurately in the valid-cue condition than in the neutral-cue condition $(p<0.01)$ and less accurately in the invalid-cue condition than in the neutral-cue condition $(p<0.01)$. Cue type did not interact with either facial feature or orientation (both $p \mathrm{~s}>0.24$ ); however, there was a significant three-way interaction between cue, feature, and orientation $\left(F_{4,120}=4.37, p=0.003\right)$. On looking at figure 4 , it appears that the effect of cueing was diminished for detecting changes to the eyes in inverted faces and the mouth in upright faces.

Only reaction times for correct trials were analysed. This resulted in a number of empty cells in the RT data owing to some subjects recording zero accuracy in some conditions. In total there were 33 missing RT values (3\% of total data cells) in thirteen different conditions, each of which was replaced with the mean for that condition. In general, the trends in the RT data followed the pattern of results found for the accuracy data; although, as is the nature of RT data, the variability was much greater and not all interactions found in the accuracy data were significant in the RT data (see figure 5).
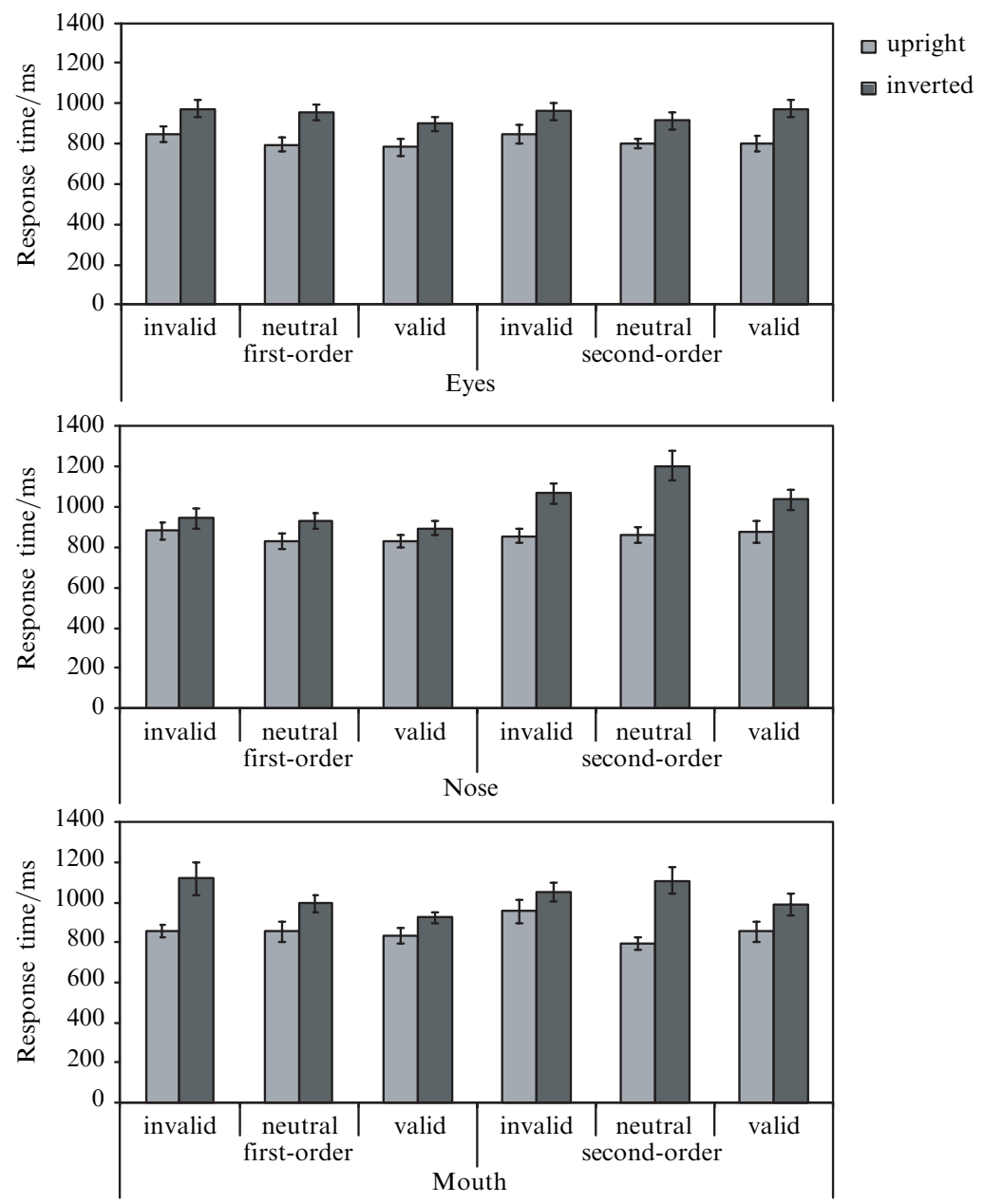

Figure 5. Results of experiment 2. Mean response times to upright and inverted faces are shown as a function of cue, change type, and orientation for each of the three facial features tested. Error bars represent standard errors of the mean. 
The four-way repeated-measures ANOVA conducted on the RT data revealed significant main effects of orientation $\left(F_{1,30}=51.74, \quad p<0.01\right.$; upright: $\mathrm{M}=911, \mathrm{SE}=10$; inverted: $\mathrm{M}=1066, \mathrm{SE}=13)$, facial feature $\left(F_{2,60}=4.38, p=0.02\right.$; eyes: $\mathrm{M}=969$, $\mathrm{SE}=14$; mouth: $\mathrm{M}=1017, \mathrm{SE}=16$; nose: $\mathrm{M}=981, \mathrm{SE}=15)$, and cue $\left(F_{2,60}=4.44\right.$, $p=0.02$; neutral cue: $\mathrm{M}=993, \mathrm{SE}=16$; valid cue: $\mathrm{M}=963, \mathrm{SE}=14$; invalid cue: $\mathrm{M}=1011, \mathrm{SE}=16)$. Although there was no main effect of change type $(p=0.10)$, the trend followed the accuracy data (first-order relations: $\mathrm{M}=976, \mathrm{SE}=12$; second-order relations: $\mathrm{M}=1002, \mathrm{SE}=13$ ).

Orientation interacted with change type $\left(F_{1,30}=6.66, p=0.02\right)$ as well as with cue $\left(F_{2,60}=4.33, p=0.02\right)$. Bonferroni adjusted a posteriori comparisons show that, while first-order and second-order changes to upright faces were detected equally fast $(p>0.05)$, first-order changes were detected faster than second-order changes in inverted faces $(p<0.05)$. That is, the effect of inversion on RT was greater for second-order than for first-order changes (see figure 5). The interaction between orientation and cue appears to be due to RT for detection of neutral-cue changes in inverted faces being slower than invalid-cue changes, while the cueing pattern for upright faces shows that neutral-cue changes were detected faster than invalid-cue changes. None of the threeway interactions or the four-way interaction was significant (all $p \mathrm{~s}>0.05$ ).

\section{General discussion}

The results of the present experiments indicate that the type of information processed is a central factor in the allocation of attention to faces. Experiment 1 used a flicker task to show (i) that detection of changes to the mouth was impaired by inversion to a greater extent than that of changes to the eyes and (ii) that inversion disrupted change detection to first-order as well as to second-order relations. Experiment 2 used valid, invalid, and neutral cues in a one-shot change-detection task. Importantly, we found that while detection of changes to first-order relations was affected by cueing, secondorder information (information thought to be 'special' to face perception) remained unaffected by cueing. Further, this cueing pattern did not depend on the facial feature involved in the change.

In both of our experimental tasks, inversion impaired detection of change to both first-order and second-order relations. Our results add to the body of research showing that not only does inversion impair processing of second-order relations between features (Friere et al 2000; Leder and Bruce 2000; Leder et al 2001; Searcy and Bartlett 1996), it also impairs the processing of first-order configural relations (Malcolm et al 2004). The magnitude of the impairment is consistently larger for second-order than for first-order relations, suggesting a particularly heavy dependence on second-order configural relations in the processing of upright faces, even when individuation of faces is not important. This suggests that information about the second-order relations in a face is just harder to extract when the face is inverted than when it is upright, independently of any attempt to 'recognise' the identity of the face. Davies and Hoffman (2002) and Barton et al (2003) argue that this is a consequence of inefficient allocation of attention to inverted faces and/or facial regions. However, the current study, and experiment 2 in particular, suggests that efficient allocation of attention to second-order relations (thought to be 'special' to face processing) plays a potentially more important role in face processing than regional differences in attentional allocation. First-order and second-order changes were detected in upright faces with similar accuracy and speed, and although we found that change-detection was better for eyes than for the mouth or nose, this pattern was not affected by cueing. That is, focused attention does not appear to play a part in the differential processing of facial features in this study. 
Overall, there was not a significant cost of invalid cueing, but there was a benefit of valid cueing, particularly for inverted faces and for first-order changes. That is, focused attention had an effect in those conditions (first-order relation changes) that do not involve processing thought to be 'special' to faces. There was no significant effect of cueing attention to a second-order relation change, suggesting that attention is automatically directed to this type of information in upright faces. Generally speaking, our results are in line with those of Davies and Hoffman (2002) in that we find that attention to, as well as the perception of, faces is impaired by inversion. Our results are also compatible with the main argument of Barton and colleagues (Barton et al 2001, 2003; Malcolm et al 2004) that spatial relations are a critical factor in the mechanism that processes upright faces (although we do not find an interaction of spatial encoding with regional salience in the inversion effect). The current study explicitly links and qualifies these findings.

The current findings may have implications for the expertise debate in face recognition. Gauthier and colleagues (Gauthier and Tarr 1997; Gauthier et al 1998; Tanaka and Gauthier 1997) argue that our face-processing abilities differ with those of objects because we are experts at individuating faces. In particular, Gauthier and colleagues have argued that, as expertise for an object category (eg faces or 'greebles') develops, so too does reliance on the processing of second-order relations. Previous research investigating change detection with novel objects (Keane et al 2003) has shown that changes to the categorical (first-order) relations of object parts are easier to detect than changes to the coordinate (second-order) relations of object parts. This is in line with the idea that first-order relations are important in lower or basic-level objectrecognition tasks (Cooper and Wojan 2000). While Gauthier and colleagues' research suggests that it would, it remains to be seen whether detection of second-order relation changes in familiar objects is unaffected by cueing (as was found for faces in the current study). If this were the case, it would suggest that upright faces and familiar objects are not processed in a similar manner to unfamiliar objects and inverted faces because the allocation of attention in objects for which we are expert is different to that of unfamiliar objects.

In conclusion, we have provided further evidence suggesting that the processing of upright faces is different from the processing of inverted faces because of the way in which attention is automatically directed in upright faces. In particular, the current results show that the second-order relations in an upright face automatically attract attention, even in a task that does not require individuation. Whether this allocation of attention to second-order configural processing is unique to faces is an area for future research.

Acknowledgments. We thank our reviewers in addition to Romina Palermo and Stephen Palmisano for their constructive comments on an earlier version of this paper.

\section{References}

Aginsky V, Tarr M J, 2000 "How are different properties of a scene encoded in visual memory?" Visual Cognition (Special Issue on Change Detection and Visual Memory) 7 147-162

Barton J J S, Deepak S, Malik N, 2003 "Attending to faces: change detection, familiarization, and inversion effects" Perception $3215-28$

Barton J J S, Keenan J P, Bass T, 2001 "Discrimination of spatial relations and features in faces: Effects of inversion and viewing duration" British Journal of Psychology $92527-549$

Collishaw S M, Hole G J, 2000 "Featural and configurational processes in the recognition of faces of different familiarity" Perception $29893-909$

Cooper E E, Wojan T J, 2000 "Differences in the coding of spatial relations in face identification and basic-level object recognition" Journal of Experimental Psychology: Learning, Memory, and Cognition $26470-488$

Davies T M, Hoffman D D, 2002 "Attention to faces: A change-blindness study" Perception 31 $1123-1146$ 
Diamond R, Carey S, 1986 "Why faces are and are not special: An effect of expertise" Journal of Experimental Psychology: General $115107-117$

Donnelly N, Davidoff J, 1999 "The mental representation of faces and houses: Issues concerning parts and wholes" Visual Recognition 6 319-343

Farah M J, 1996 "Is face recognition special? Evidence from neuropsychology" Behavioral Brain Research $76181-189$

Friere A, Lee K, Symons L A, 2000 "The face-inversion effect as a deficit in the encoding of configural information: Direct evidence" Perception $29159-170$

Gauthier I, Tarr M J, 1997 "Becoming a 'Greeble' expert: Exploring mechanisms for face recognition" Vision Research $371673-1682$

Gauthier I, Tarr M J, Anderson A W, Skudlarski P, Gore J C, 1999 "Activation of the middle fusiform 'face area' increases with expertise in recognizing novel objects" Nature Neuroscience $2568-573$

Gauthier I, Williams P, Tarr M J, Tanaka J, 1998 "Training 'Greeble' experts: A framework for studying expert object recognition processes" Vision Research (Special Issue on Models of Recognition) $382401-2428$

Gosselin F, Schyns P G, 2001 "Bubbles: a technique to reveal the use of information in recognition tasks" Vision Research 412261 -2271

Haig N D, 1984 "The effect of feature displacement on face recognition" Perception 13 505-512

Hayes A, 1988 "Identification of two-tone images; some implications for high- and low-spatialfrequency processes in human vision" Perception $17429-436$

Hosie J A, Ellis H D, Haig N D, 1988 "The effect of feature displacement on the perception of well-known faces" Perception $17461-474$

Keane S K, Hayward W G, Burke D, 2003 "Detection of three types of changes to novel objects" Visual Cognition $10101-127$

Kemp R, McManus C, Pigott T, 1990 "Sensitivity to the displacement of facial features in negative and inverted images" Perception 19531 - 543

Leder H, Bruce V, 2000 "When inverted faces are recognized: the role of configural information in face recognition" Quarterly Journal of Experimental Psychology A 53 513-536

Leder H, Candrian G, Huber O, Bruce V, 2001 "Configural features in the context of upright and inverted faces" Perception $3073-83$

Malcolm G L, Leung C, Barton J J S, 2004 "Regional variation in the inversion effect for faces: Differential effects for feature shape, feature configuration, and external contour" Perception $331221-1231$

Maurer D, Le Grand R, Mondloch C J, 2002 "The many faces of configural processing" Trends in Cognitive Sciences $6255-260$

Palermo R, Rhodes G, 2007 "Are you always on my mind? A review of how face perception and attention interact" Neuropsychologia $4575-92$

Posner M I, Nissen M J, Ogden W C, 1978 "Attended and unattended processing modes: The role of set for spatial location", in Modes of Perceiving and Processing Information Eds H L Pick, I J Saltzman (Hillsdale, NJ: Lawrence Erlbaum Associates) pp 137-158

Rensink R A, 2000 "The dynamic representation of scenes" Visual Cognition 7 17-42

Rensink R A, 2002 "Change detection” Annual Review of Psychology 53 254-277

Rensink R A, O'Regan J K, Clark J J, 1997 "To see or not to see: The need for attention to perceive changes in scenes" Psychological Science 8368 - 373

Rensink R A, O'Regan J K, Clark J J, 2000 "On the failure to detect changes in scenes across brief interruptions" Visual Cognition (Special Issue on Change Detection and Visual Memory) $7127-145$

Schwaninger A, Lobmaier J S, Collishaw S M, 2002 "Role of featural and configural information in familiar and unfamiliar face recognition" Lecture Notes in Computer Science $\mathbf{2 5 2 5}$ $643-650$

Searcy J H, Bartlett J C, 1996 "Inversion and processing of component and spatial-relational information in faces" Journal of Experimental Psychology: Human Perception and Performance $22904-915$

Sergent J, 1984 "An investigation into component and configural processes underlying face recognition" British Journal of Psychology 75221 - 242

Sergent J, 1986 "Microgenesis of face perception", in Aspects of Face Processing Eds H D Ellis, M A Jeeves, F Newcombe, A Young (Dordrecht: Martinus Nijhoff) pp 17-33

Shepherd J, Davies G, Ellis H, 1981 "Studies of cue saliency", in Perceiving and Remembering Faces Eds G Davies, H Ellis, J Shepherd (London: Academic Press) pp 105-131 
Tanaka J W, Gauthier I, 1997 "Expertise in object and face recognition", in Psychology of Learning and Motivation volume 36, Eds R L Goldstone, P G Schyns, D L Medin (San Diego, CA: Academic Press) pp $83-125$

Thompson P, 1980 "Margaret Thatcher: a new illusion" Perception $9483-484$

Wolfe J M, 1999 "Inattentional amnesia", in Fleeting Memories Ed. V Coltheart (Cambridge, MA: MIT Press) pp $71-94$ 


\section{PERTEPTION}

VOLUME 362007

www.perceptionweb.com

Conditions of use. This article may be downloaded from the Perception website for personal research by members of subscribing organisations. Authors are entitled to distribute their own article (in printed form or by e-mail) to up to 50 people. This PDF may not be placed on any website (or other online distribution system) without permission of the publisher. 